\title{
The economic burden of prematurity in Canada
}

\author{
Karissa M Johnston' ${ }^{1}$, Katherine Gooch², Ellen Korol', Pamela Vo², Oghenowede Eyawo ${ }^{1,3}$, Pamela Bradt ${ }^{4}$ \\ and Adrian Levy ${ }^{1,5^{*}}$
}

\begin{abstract}
Background: Preterm birth is a major risk factor for morbidity and mortality among infants worldwide, and imposes considerable burden on health, education and social services, as well as on families and caregivers. Morbidity and mortality resulting from preterm birth is highest among early ( $<28$ weeks gestational age) and moderate (28-32 weeks) preterm infants, relative to late preterm infants (33-36 weeks). However, substantial societal burden is associated with late prematurity due to the larger number of late preterm infants relative to early and moderate preterm infants.

Methods: The aim in this study was to characterize the burden of premature birth in Canada for early, moderate, and late premature infants, including resource utilization, direct medical costs, parental out-of-pocket costs, education costs, and mortality, using a validated and published decision model from the UK, and adapting it to a Canadian setting based on analysis of administrative, population-based data from Québec.
\end{abstract}

Results: Two-year survival was estimated at 56.0\% for early preterm infants, $92.8 \%$ for moderate preterm infants, and 98.4\% for late preterm infants. Per infant resource utilization consistently decreased with age. For moderately preterm infants, hospital days ranged from 1.6 at age two to 0.09 at age ten. Cost per infant over the first ten years of life was estimated to be $\$ 67,467$ for early preterm infants, $\$ 52,796$ for moderate preterm infants, and $\$ 10,010$ for late preterm infants. Based on population sizes this corresponds to total national costs of $\$ 123.3$ million for early preterm infants, \$255.6 million for moderate preterm infants, \$208.2 million for late preterm infants, and \$587.1 million for all infants.

Conclusion: Premature birth results in significant infant morbidity, mortality, healthcare utilization and costs in Canada. A comprehensive decision-model based on analysis of a Canadian population-based administrative data source suggested that the greatest national-level burden is associated with moderate preterm infants due to both a large cost per infant and population size while the highest individual-level burden is in early preterm infants and the largest total population size is in late preterm infants. Although the highest medical costs are incurred during the neonatal period, greater resource utilization and costs extend into childhood.

\section{Background}

Preterm birth, defined as birth before the completion of 37 weeks gestation, [1] is a major risk factor for morbidity and mortality among infants worldwide, and imposes considerable burden on health, education and social services, as well as on families and caregivers [1-5]. The epidemiologic burden of prematurity in Canada is substantial; approximately eight percent of live in-hospital births in 2009-2010 were preterm; [6,7] and considerably high hospital costs and other health expenditures have been reported for this population.

\footnotetext{
* Correspondence: adrian.levy@dal.ca

'Epidemiology, Oxford Outcomes Ltd., Vancouver, Canada

${ }^{5}$ Department of Community Health \& Epidemiology, Dalhousie University,

5790 University Ave., Halifax, Nova Scotia B3H 1V7, Canada

Full list of author information is available at the end of the article
}

Morbidity and mortality resulting from preterm birth is highest among early (born at less than 28 weeks gestational age) and moderate (born between 28 and 32 weeks gestational age) preterm infants $[8,9]$. The morbidity impact of preterm birth is not limited to the neonatal period, but also extends into later periods in life resulting in cognitive developmental impairments, learning difficulties, social and behavioral problems $[8,10,11]$. Learning disability is associated with considerable costs to individuals, families, and the society [12]. The epidemiology, causes and outcomes of preterm birth have been extensively reviewed $[2,8,10,13]$. Due to the underdeveloped lung tissue, respiratory morbidity is commonly associated with prematurity. Less common prematurity-associated morbidities include sepsis, intraventricular hemorrhage, periventricular leukomalacia, necrotizing enterocolitis, cerebral palsy,

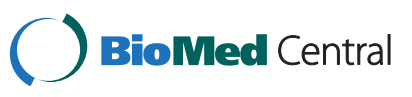


retinopathy of prematurity $[14,15]$. Preterm infants have been shown to have higher rates of childhood hospitalization compared to infants born closer to term $[16,17]$.

The primary objective of this study was to characterize the burden of prematurity in Canada over the first ten years of life-as characterized by healthcare resource utilization, direct medical costs, indirect costs associated with lost productivity, and mortality-to describe trends in utilization patterns from infancy and into childhood, and across gestational-age categories. These costs are characterized both as cost per individual preterm infant, and scaled to the Canadian population level by extrapolating individual costs to the number of preterm infants born each year in Canada, and the corresponding gestational age distribution.

\section{Methods}

Data from longitudinal, administrative population-based databases from Québec, Canada were used to meet this objective. The methodology presents a Canadian adaptation of a previously developed burden of illness model from the United Kingdom (UK) estimating the long-term costs of preterm birth throughout childhood in England and Wales, [13] based on the incorporation of population-based empirical resource utilization data from Québec. Consistent with other recent studies, [18] we assumed that the population-based Québec data were generalizable to the Canadian population, and the overall economic burden of prematurity in Canada was estimated using a Markov decision model. The model structure is shown in Figure 1. Infants entering the model were stratified by gestational age at birth, with early preterm defined as $<28$ weeks, moderate preterm defined as $28-32$ weeks, and late preterm defined as 33-36 weeks [13]. Costs were included from the time of prenatal care through the first ten years of life for surviving preterm infants. Overall components of costs included in the model were medical costs (for both the infant and excess prenatal costs for the mother) and indirect costs associated with lost productivity for parents. Costs of education, additional prenatal care, and of building neonatal facilities were considered in sensitivity analysis.

\section{Data source}

Resource utilization parameters were populated using Régie de l'assurance maladie du Québec (RAMQ) physician billing data from Québec, Canada linked to MED-ÉCHO hospital discharge abstract databases. A retrospective population-based design was used to establish and follow a birth cohort of all premature infants born during 19961997 until age ten. The RAMQ insures all provincial health plan registrants in Québec (99\% of 7,731,600 Québec residents in 2006) for necessary medical and hospital services and their databases include: 1) claims $[19,20]$ from the approximately 92\% of Québec physicians who work on a fee-for-service basis, [20] and 2) all acute care hospital discharge abstracts in the province. Ethical approval of the protocol and data release was provided by the Commission d'acces à l'information du Québec. The Markov model used to estimate the economic burden of prematurity in Canada, adapted from an alogous model developed for the the UK, was developed in Microsoft Excel. Data from the RAMQ was stored in a SQL database (Microsoft SQL version 10.50.1600.1), and analysis was conducted using R 2.13.1.

\section{Model structure}

Epidemiological and resource utilization parameters were stratified by gestational age category, and overall results are a weighted average of gestational age-specific results and cost parameters, based on the relative distributions of early, moderate, and late preterm infants. Following live birth, infants who did not die in the delivery room went on to either admission to a neonatal care facility or discharge directly home. Following hospital discharge, a single model state was used to describe time until age two to account for increased medical costs incurred during early childhood. Following age two, costs were accrued annually until age ten. Level of disability was incorporated in a sensitivity analysis to characterize costs associated with special education requirements. Disabilities included motor function (including cerebral palsy), vision and hearing impairment, and cognitive abilities, consistent with the definitions used within the VICSG cohort [21]. Children were eligible to shift across disability states over time based on a Markov model structure, in which the probability of entering a disability state in a given year was dependent solely on the current disability state. At age two, the distribution across disability levels was based on gestational age at birth; in subsequent years, a Markov transition model was used to describe shifts in disability levels over time [13]. Between ages two and ten, medical costs were accrued annually based on observed resource utilization and costs by gestational age category from the RAMQ data.

The probability of live discharge from the neonatal intensive care unit, by gestational age, were taken from a study by the Canadian NICU Network during 1996-1997 [22]. Additional parameters describing survival probabilities from birth to age ten, and trajectories of disability over time were taken from a published decision model, [13] with the exception of the gestational-age specific probability of death in the delivery room or in the neonatal intensive care unit, which were taken from a more recent publication based on a population-based study of all births in New South Wales and Australian Capital Territory in Australia [23]. The RAMQ data did not contain sufficient information to compute all survival-related parameters for 


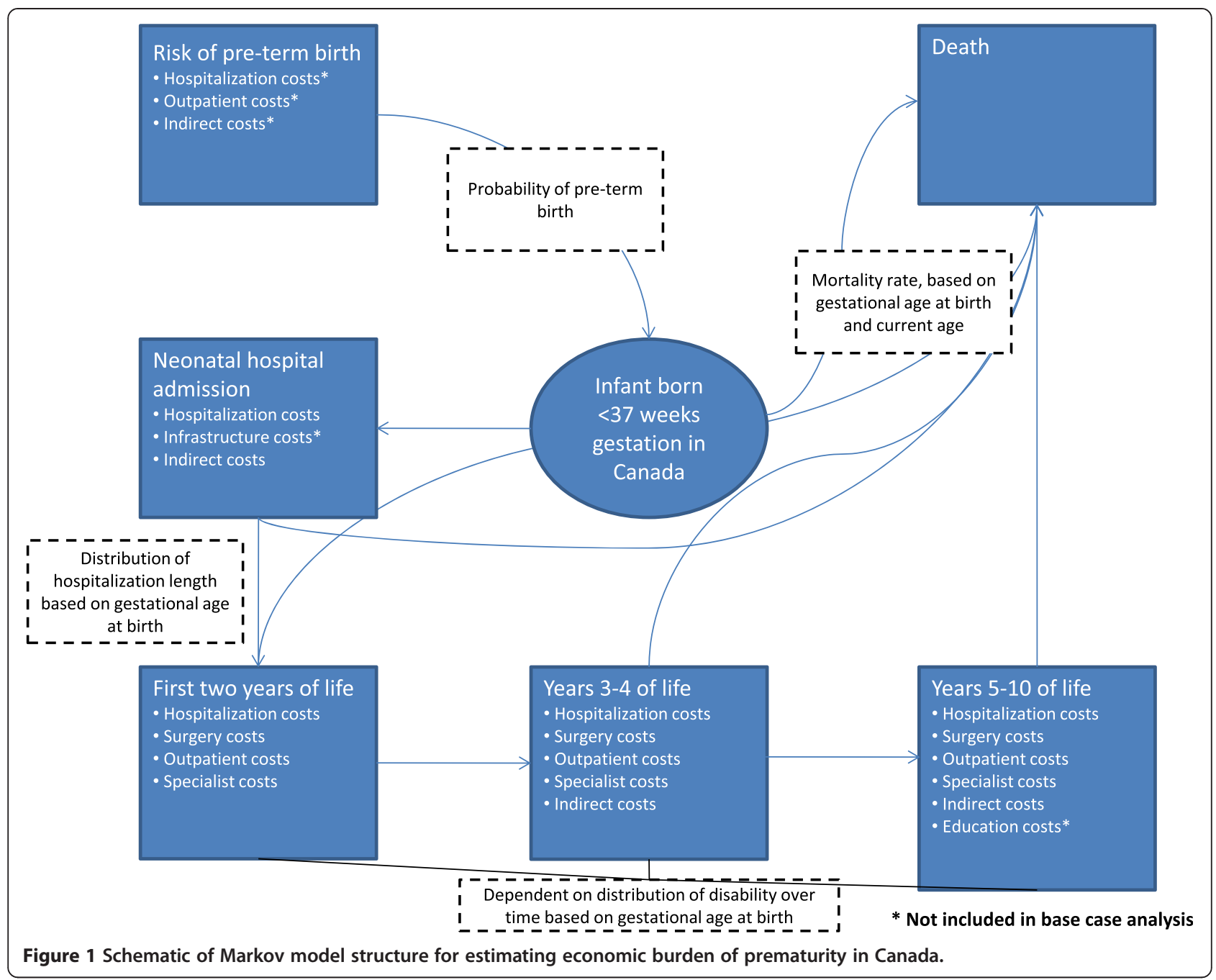

Canada, but where available they were computed and found to be comparable to those calculated for the UK and Australia [23-25].

Costs were discounted at $5 \%$ annually.

\section{Additional costs considered in sensitivity analysis}

Several additional costs associated with preterm birth were considered in exploratory sensitivity analysis. The primary analysis did not incorporate these elements because empirical data were not available to calculate the relevant parameters, which were instead estimated based on assumption and expert opinion.

Excess prenatal costs were included in addition to costs associated with the infant following birth. These costs were defined as those associated with additional resource utilization incurred by women identified as high-risk for preterm labor and were based on published sources and expert opinion (Additional file 1: Table S1). In absence of published literature, clinical expert consultation was sought and it was assumed that $50 \%$ of preterm births were associated with excess prenatal costs, and the remaining $50 \%$ of preterm births were not identified in pregnancy and as such were not associated with excess prenatal resource utilization. Education costs associated with special education requirements for children with disability included from age five onwards. The additional contribution of infrastructure cost to neonatal facility perdiem costs was based on the assumption that a neonatal facility would cost $\$ 2.5$ million to build, would contain 25 infant-beds, and would have an effective lifetime of 30 years $[26,27]$. Empirical data were not available for these parameters. This resulted in an additional cost of $\$ 18.26$ per infant per day associated with neonatal care [28].

\section{Cost parameters}

Model parameters associated with resource utilization, the epidemiology of preterm birth, and Markov model transition probabilities were taken from a published model [13]. 
National-level costs were based on an assumption of 380,863 live births in Canada, [29] with $0.40 \%$ of births early preterm, $1.14 \%$ moderate preterm, and $6.19 \%$ late preterm [30].

Unit costs were based on Ontario 2012 costs; the most recent available costs were taken from published sources and were inflated as needed to 2012 values as needed using inflation indices based on the Statistics Canada Consumer Price Index (Additional file 1: Table S2). Unit costs used within the model are given in Table 1. The average unit cost of $\$ 111.88$ associated with in-hospital procedures was calculated by multiplying unit costs from the 2012 Ontario schedule of physician benefits [31] to the ten most commonly listed procedures within the RAMQ data.

Indirect costs associated with parental time taken off of work to attend any medical visits and hospitalizations incurred by their child were included. It was assumed that these costs would be incurred from ages two onwards, and that premature infants would have a full-time caregiver available for medical appointments and hospitalizations from discharge until age two. From age two onward, indirect costs due to lost productivity were calculated, stratified by gestational age category, based on the number of outpatient visits and inpatient days observed in the RAMQ database. It was assumed that outpatient visits would be associated with two hours taken off work and that inpatient days would be associated with eight hours taken off work, and assumed an hourly wage of $\$ 23.18$, based on a full-time female employee.

\section{Resource utilization parameters}

The linked RAMQ and MED-ÉCHO databases were used to extract the following resource utilization and cost parameters, stratified by gestational age category and current age: number of hospital days, stratified into general ward and intensive care unit; surgeries and other procedures received in hospital; and outpatient costs billed by the physician. Data were extracted from 1996 to 2007 inclusive, for all preterm infants born in 1996 and 1997. Infants were excluded from the analysis if no subsequent medical or hospital visits occurred after the initial birth hospitalization and no record of death could be found. Infants were also excluded if a transfer to another hospital during the initial birth hospitalization was recorded, due to inconsistencies in the data associated with these entries. In estimating cumulative costs throughout childhood within the decision model, agespecific costs for each gestational age category were weighted by the proportion that an infant would survive to that age. The percentage distribution of utilization of mainstream primary education and special education by disability level, considered in sensitivity analysis, is given in Additional file 1: Table S3.

\section{Canadian resource utilization}

Average resource utilization and costs per child were extrapolated to Canadian estimates by multiplying costs by the estimated number of live births [29] and the proportion of premature births in Canada [30] - both overall prematurity and stratified by gestational age category.

\section{Probabilistic sensitivity analysis}

A probabilistic sensitivity analysis (PSA) was undertaken to assess the impact of uncertainty in model input parameters on potential variability of overall total cost results. The epidemiological parameters that were assumed to be consistent with those reported previously were assumed to follow the described distributions [25]. For de novo Canadian resource utilization and cost parameters, standard errors were estimated directly from the RAMQ data, and normal distributions were assumed.

\section{Results}

The distributions of survival and disability at ages two and ten, respectively, stratified by gestational age at birth, are shown in Figure 2. Based on clinical input parameters, model projections estimated that the survival rate amongst live births at age two would be $56.0 \%$ of early preterm infants, $92.8 \%$ of moderate preterm infants, and $98.4 \%$ of late preterm infants. The corresponding survival rates at age ten were $55.9 \%$ for early preterm, $92.6 \%$ for moderate preterm, and $98.2 \%$ for late preterm, reflecting the small mortality rates between age two and age ten for all gestational age categories. Compared to survival, there was greater variation in distribution of disability between age two and age ten. For moderate and late preterm babies there was a shift from no and mild disability to moderate and severe disability, although the majority of children remained in the no disability state at age ten. For early preterm babies, the relative proportion of severe disability was greater at age two, with a small shift from moderate and severe disability to no disability and mild disability at age ten.

Figure 3 describes resource utilization (hospitalizations, hospital days, inpatient interventions, intensive care unit visits, and outpatient physician visits) from birth to age ten, separated by gestational age category. Total inpatient days and outpatient costs are reported in Table 2. All resource utilization notably decreased with age across all gestational age categories. For moderately preterm infants, hospital days ranged from 1.6 days at age two to 0.09 days at age ten. Costs associated with outpatient visits for moderately preterm infants ranged from $\$ 1,453$ prior to age two to $\$ 123$ at age ten. Resource utilization tended to be similar between early and moderate preterm infants, and higher for these categories compared to late preterm infants. In these analyses, results at each age are specific to the subset of individuals who survived until that age, 
Table 1 Unit costs used associated with the burden of prematurity in Canada

\begin{tabular}{|c|c|c|}
\hline Item & Unit cost (\$CAD) & Source/comments \\
\hline \multicolumn{3}{|c|}{ Prenatal unit costs for women at risk of preterm labor* } \\
\hline Inpatient days & 809.87 & Canadian Institute of Health Information [7] \\
\hline Additional midwife visits & 23.20 & Working in Canada [32] \\
\hline Additional obstetrician visits & 101.70 & OHIP Schedule of benefits [30] \\
\hline Cervical cerclage & 145.10 & OHIP Schedule of benefits [30] \\
\hline Beta agonists & 6.00 & Ontario Drug Benefit formulary [33] \\
\hline Oxytocin receptor antagonists & 67.75 & OHIP Schedule of benefits [30] \\
\hline \multicolumn{3}{|l|}{ Unit costs for different modes of delivery* } \\
\hline Spontaneous delivery & $498.70^{* * *}$ & OHIP Schedule of benefits [30] \\
\hline Instrumental delivery & $625.66^{* * *}$ & OHIP Schedule of benefits [30] \\
\hline Elective caesarean & $757.11^{* * *}$ & OHIP Schedule of benefits [30] \\
\hline Emergency caesarean & $786.51^{* * *}$ & OHIP Schedule of benefits [30] \\
\hline \multicolumn{3}{|c|}{ Unit costs associated with neonatal intensive care unit for preterm infants* } \\
\hline Neonatal intensive care & $1,628.60$ & $\mathrm{OCCl}[34]$ \\
\hline Neonatal normal care & 388.00 & $\mathrm{OCCl}[34]$ \\
\hline \multicolumn{3}{|c|}{ Parameters associated with neonatal intensive care unit infrastructure } \\
\hline Cost of building a facility & $2,500,000$ & Assumption \\
\hline Number of infants cared for simultaneously & 25 & Assumption \\
\hline Lifetime of facility (years) & 30 & Assumption \\
\hline Resulting cost per infant per day & 9.13 & Function of above parameters \\
\hline \multicolumn{3}{|c|}{ Unit costs between hospital discharge and age 2 years for preterm infants } \\
\hline Inpatient stay (per day) & 628.49 & Harris 2011 [35] \\
\hline Outpatient visits & NA & Physician billings per visit used directly from RAMQ \\
\hline \multicolumn{3}{|c|}{ Unit costs incurred between age 2 and 10 years for preterm infants* } \\
\hline Pediatric ICU stay (per day) & $2,002.86^{* * *}$ & Harris 2011 [35] \\
\hline Pediatric procedures & $111.88^{*}$ & RAMQ and OCCI [34] \\
\hline Other pediatric inpatient stay (per day) & 628.49 & Harris 2011 [35] \\
\hline Outpatient visits & NA & Physician billings per visit used directly from RAMQ \\
\hline Mainstream primary school & $7,720.05^{*}$ & Learning Disabilities in Canada Report [12] \\
\hline Special school & $15,666.45^{*}$ & Learning Disabilities in Canada Report [12] \\
\hline \multicolumn{3}{|c|}{ Indirect costs incurred by families of preterm infants } \\
\hline Gross pay per hour (male, full-time) & 26.33 & Statistics Canada (June 2012) [36] \\
\hline Gross pay per hour (female, full-time) & 23.18 & Statistics Canada (June 2012) [36] \\
\hline Gross pay per hour (male, part-time) & 15.62 & Statistics Canada (June 2012) [36] \\
\hline Gross pay per hour (female, part-time) & 17.36 & Statistics Canada (June 2012) [36] \\
\hline
\end{tabular}

${ }^{*}$ Costs were inflated to 2012 where appropriate by using Canadian health inflators; ${ }^{* * *}$ Weighted average inflated to 2012 ; ClHI = Canadian Institute for Health Information; $\mathrm{OCCl}=$ Ontario Case Costing Initiative; OHIP = Ontario Health Insurance Plan; RAMQ = Regie de l'assurance Maladie du Quebec.

i.e. for any given age group, all resource utilization analyses were based on a denominator of surviving infants, and infants who died prior to that age were excluded from analysis. When calculating resource utilization from the RAMQ database, it was assumed that early and moderate preterm infants with no record of neonatal hospitalization died prior to admission or were otherwise lost to follow up and were excluded, while late preterm infants with no record of neonatal hospitalization were assumed to have been discharged directly home.

The total economic burden of prematurity by category of expenditure (neonatal costs,direct medical costs in subsequent years, and lost productivity costs) is reported in Table 3, both aggregated over all preterm infants and stratified by gestational age at birth. Conversely to the above-described analyses in Figure 3, costs per infant were 


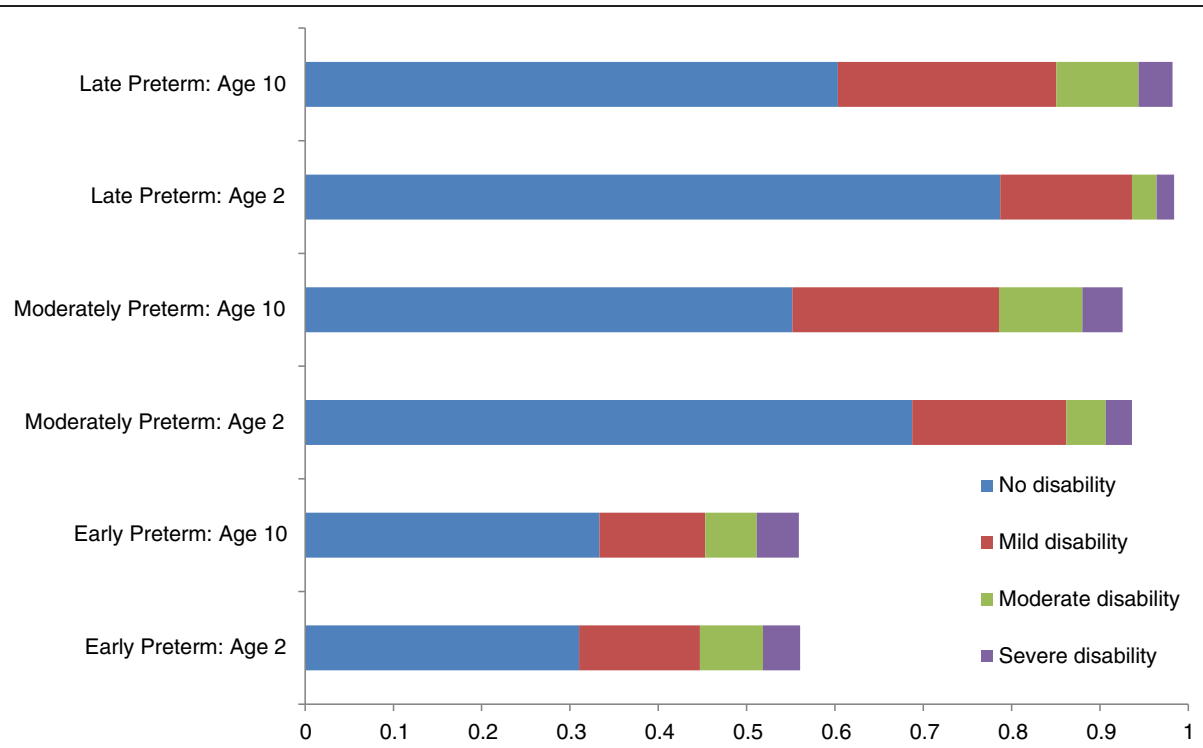

Figure 2 Distribution of Canadian live births across disability levels for preterm infants by gestational age.

averaged over all live births, including those who died during infancy or childhood, and costs throughout childhood are downweighted as applicable to reflect the smaller surviving population size at each year of life. Cost per infant over the first ten years of life was estimated to be $\$ 67,467$ (PSA 2.5th-97.5th percentiles: $\$ 52,796-\$ 83,206$ ) for early preterm infants, $\$ 54,554$ (PSA 2.5 th-97.5th percentiles: $\$ 46,301-\$ 66,422)$ for moderate preterm infants, and $\$ 10,010$ (PSA 2.5th-97.5th percentiles: $\$ 8,649-\$ 13,296$ ) for late preterm infants. Based on population sizes this corresponds to total national costs of $\$ 123.3$ million for early preterm infants, $\$ 255.6$ million for moderate preterm
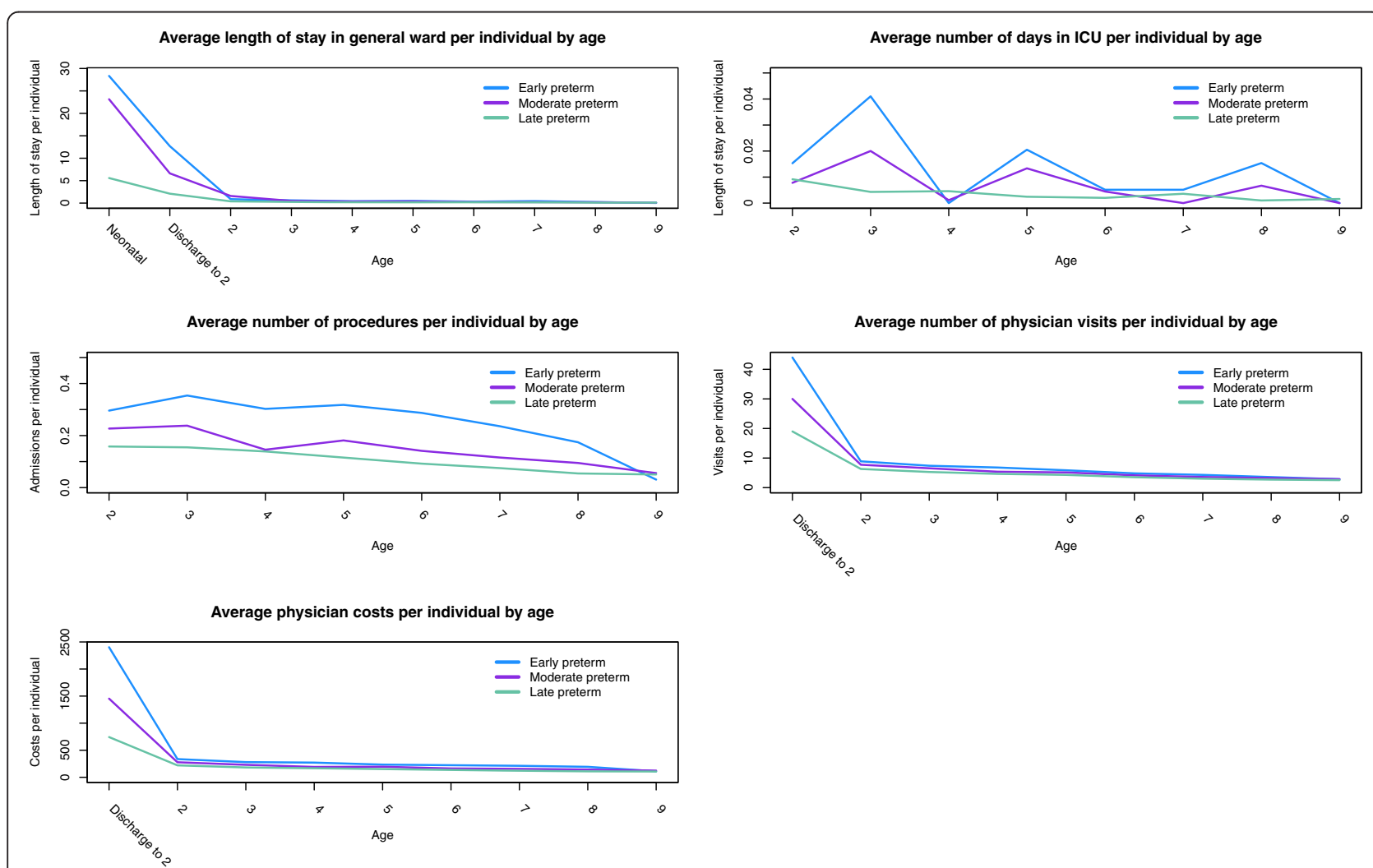

Figure 3 Resource use per individual in the Québec cohort from birth to age ten. 
Table 2 Average number and associated standard error of inpatient hospital days, outpatient costs, and associated indirect costs incurred due to lost productivity by caregivers of preterm infants from age 2-10, stratified by gestational age at birth and current age

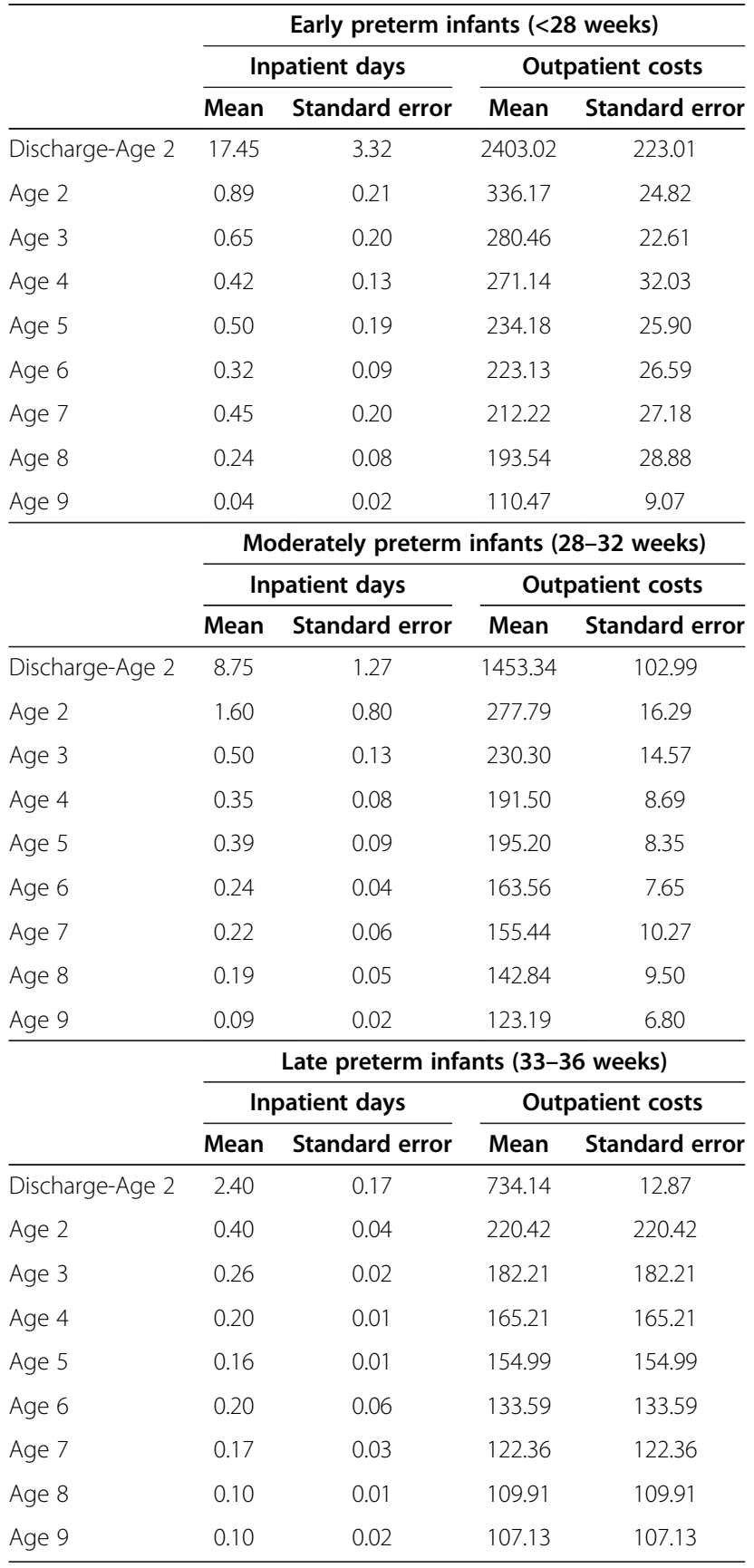

infants, \$208.2 million for late preterm infants, and \$587.1 million for all infants. While individual-costs per infant were highest for moderately preterm infants, national-level costs were greater for moderate and late preterm infants due to the larger population size.

At the individual level, for all gestational age categories, the largest contributor to total costs was the cost associated with the neonatal intensive care unit stay, followed by medical costs incurred between discharge and age two. Across categories of expenditure, individual-level costs tended to be highest for early preterm infants prior to age two, followed by moderate preterm infants and late preterm infants. Prior to age two, costs were similar between moderate and preterm infants, and substantially lower for late preterm infants. The most substantial cost differences were in neonatal hospitalization which ranged from $\$ 3,768$ in late preterm infants to $\$ 53,308$ in early preterm infants. After age two, costs were comparable across all age categories, although this implies that costs incurred by surviving children were highest for early and moderate preterms as the denominator was all live births, and there was notably higher mortality following live births for the earlier gestational age categories.

\section{Discussion}

In this study, a decision model was used to capture trends in survival, resource utilization, and indirect costs over the first ten years of life for preterm infants in Canada. A rigorous and comprehensive decision model, originally developed for the UK, was adapted to the Canadian setting by updating unit costs to Canadian values, and quantifying resource utilization by age and gestational age at birth category using a population-based real-world administrative data source. The results of this study allow for potential interventions to delay or prevent preterm birth, or to prevent morbidity in preterm infants to be contextualized with respect to the overall burden.

A recent study published by Landry et al. reported resource utilization for infants born in Québec from 1983-1992, although this was restricted to infants with respiratory complications, and utilization and costs were not further stratified by gestational age at birth [18]. The study describes here includes resource utilization and associated costs for all preterm infants born during 1996-1997, regardless of specific complications, and all results are stratified by gestational age at birth. Total medical costs were higher in the Landry et al. study, $\$ 10,719-\$ 13,472$ per person-year across respiratory distress syndrome (RDS) and bronchopulmonary dysplasia (BPD) complications, respectively, compared to $\$ 22,794$ over ten years estimated hear as a weighted average of direct medical costs and lost productivity costs over the first ten years of life. This discrepancy is explained in part by the restriction in the Landry et al. study to infants with RDS and/or BPD, who would be expected to incur greater resource utilization and costs as a result of these comorbidities. In addition, in the study reported here, the denominator was all live births, such that infants who died during the ten-year follow-up period would only contribute resource utilization and costs until time of death; this is particularly notable for extremely preterm 
Table 3 Individual and national economic burden of prematurity in Canada (\$CAD), stratified by gestational age

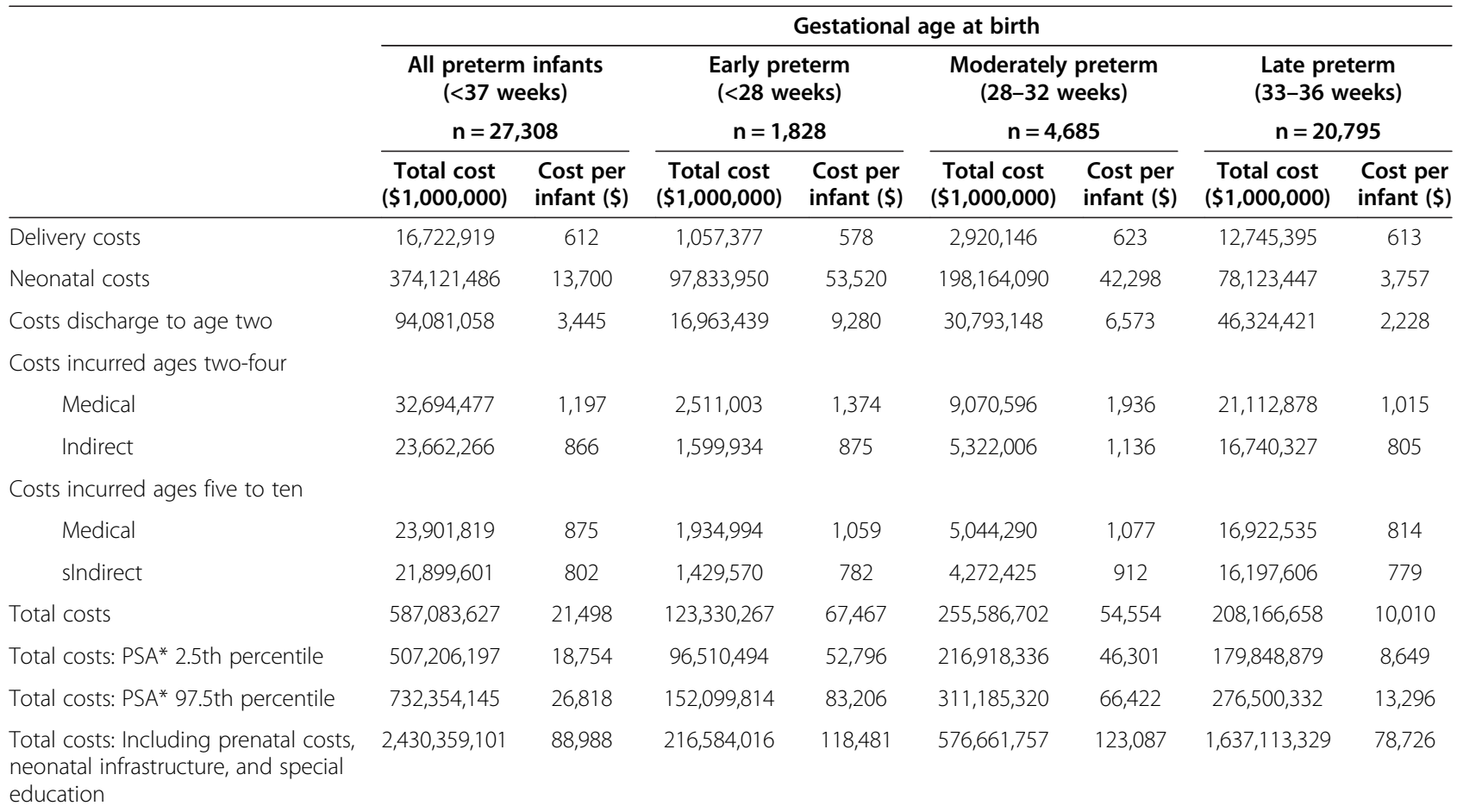

*PSA = Probabilistic sensitivity analysis.

infants, for whom over $40 \%$ were estimated to die prior to age two. While Landry et al. considered pharmaceutical costs, and this study did not, this is not anticipated to be a major source of discrepancy as they comprised a relatively minor proportion of overall medical costs (approximately $1-2 \%)[18]$.

Consistent with the medical literature, a dramatic improvement in survival in moderate and late preterm infants relative to early preterm infants was observed. Not surprisingly, neonatal intensive care costs were the largest contributor to overall medical costs amongst all preterm infants. Neonatal costs associated with moderate preterm infants were found to be similar to early preterm infants. When considered in exploratory sensitivity analysis, education costs were an important cost driver, and were highest in late preterm infants, due to the larger number of survivors and the larger proportion attending a mainstream primary school, relative to earlier preterm categories with higher prevalence of severe disability, associated with not attending a mainstream school (Additional file 1: Table S3).

The general trend in overall costs was that early and moderate preterm infants tended to incur similar costs, with much lower costs observed in late preterm infants. Based on model structure, there are two main determinants of costs incurred: the proportion surviving and the level of resource utilization incurred by survivors. Amongst surviving infants, early preterm infants tended to have the greatest medical resource utilization amongst all categories of utilization and at all ages (Figure 3). However, these infants also experienced the lowest survival rates, such that a smaller proportion of live-born preterm infants survive into childhood and incur related costs (Figure 2). Thus, the similar cost per infant for moderate preterm infants relative to early preterm infants is reflective of the higher survival rate in moderate preterm infants which results in a greater proportion of infants incurring costs throughout childhood.

A key strength of this study is the high quality and comprehensive nature of the data used to populate model parameters. The RAMQ data describe populationbased resource utilization for all preterm infants born in the province of Québec during 1996 and 1997 over their first ten years of life. These resource utilization data were combined with a published model describing the epidemiology, survival, and disability trajectories of preterm infants, and unit costs for health resources were updated to 2012 values using inflation factors. Thus, the Canadian adaptation of the model provides an up to date and comprehensive estimate of the overall economic burden of prematurity in Canada. In addition, these results are potentially generalizable beyond Canada to countries with similar trends in pediatric treatment patterns and relative costs of health resources.

Limitations to the approach include the fact that prescription medication costs were excluded from the 
analysis as the RAMQ data only include prescription records for a subset of individuals with medication coverage. The assumption was made that for preterm infants during childhood, the costs associated with medications would be substantially less important than those associated with hospitalizations and outpatient visits. In addition, in order to maximize length of available follow-up, the analysis was based on a cohort of infants born during 1996-1997, and, as such, their patterns of care may not reflect current treatment practices. Results were scaled to the population of Canada based on the assumption that clinical outcomes and resource utilization in Québec, and unit costs for medical resources from Ontario would be generalizable to the rest of Canada. While health care in Canada is delivered at the provincial level, it was assumed that individual provinces would not vary substantially with respect to pediatric clinical outcomes, resource utilization, and unit costs, such that the observed values for Québec and Ontario could serve as a suitable approximation for other provinces. Ontario, the largest Canadian province was selected as the most applicable province for selecting unit costs. While model parameters associated with survival and long-term disability trajectory were taken from UK and Australian sources where not available for Canada, where values were available for multiple sources (e.g. probabilities of live discharge from hospital for gestational age 23-35 weeks were available for both Canada [22] and the UK, [25] while probabilities of death in the delivery room for gestational ages 23-31 weeks were available for both Australia [23] and the UK [25]), they were compared and found to be comparable, supporting the generalizability of such parameters across health care systems. This is further supported by an international comparison of perinatal and infant mortality statistics, in which similar results were reported for Canada, the UK, and Australia (Tables 2-4 of reference) [24].

The strength of an economic model is dictated by the strength of evidence used as model inputs. The primary, core analysis was based on inputs for which empirical data were available, including the incidence of premature birth and gestational age distribution in Canada, and survival and resource utilization and costs associated with preterm infants in Québec. While the highest quality evidence available was used in the primary analysis and sensitivity analyses, where empirical evidence was lacking, expert opinion evidence was used as sensitivity analysis, for prenatal resource utilization, special education associated with disability, and construction of neonatal facilities. For the inclusion of excess healthcare utilization for prenatal care, it was assumed that $50 \%$ of preterm births would have been associated with such care, due to a paucity of published estimates. In adapting the model to a Canadian setting, it was assumed that the values assumed within the UK model describing distribution of disability, and requirements for special needs education would be relevant for Canada.

Future extensions of this work include the assessment of temporal trends in care to project expected updates to utilization estimates, and to compare the costs of preterm infants to those incurred by full-term infants in order to estimate an incremental cost of prematurity in addition to the absolute costs presented here. In addition, it would be of interest to expand the burden of illness model to compare differences in economic burden with respect to specific medical conditions relative to prematurity and pediatric populations, such as respiratory morbidity. Finally, the incorporation of quality-of-life estimation and empirical estimation of out-of-pocket expenses and lost productivity costs in Canadian families in addition to survival, resource utilization, and economic outcomes could provide a more inclusive view of the burden of prematurity throughout childhood, and would allow for a more comprehensive comparison of the overall burden experienced during childhood by preterm infants born at varying gestational ages. The model described here allows for numerous "what if" scenarios to be considered in future consideration of additional research questions.

\section{Conclusion}

Premature birth results in significant infant morbidity, mortality, healthcare utilization and costs in Canada. The results of this study allow for potential interventions to delay or prevent preterm birth, or to prevent morbidity in preterm infants to be contextualized with respect to the overall burden. A comprehensive decision-model based on analysis of a Canadian population-based Canadian administrative data source suggested that substantial costs per infant are observed in early and moderate preterm infants, but when scaled to the national level, late preterm infants contribute a substantial burden due to the relatively larger population size. Although the highest medical costs are incurred during the neonatal period, higher resource utilization and costs extend into childhood.

\section{Additional file}

Additional file 1: Supplementary data input tables.

\section{Competing interests}

P. Vo is an AbbVie Inc employee and may hold stock or options in AbbVie Inc. K. Gooch is an AbbVie Inc employee and may hold stock or options in AbbVie Inc.

This study was funded by AbbVie, North Chicago, IL.

\section{Authors' contributions}

KG developed study objectives and reviewed and provided significant feedback on the manuscript. KMJ designed and built the model, and wrote the first draft of the manuscript. EK performed statistical analysis of RAMQ data. EO contributed to literature review, and manuscript writing. AL and PV provided significant feedback on the manuscript. All authors read and approved the final manuscript. 


\section{Acknowledgements}

The authors wish to acknowledge Sarah Goring, Andrew Laws, and Meagan Bibby for contributing to the modelling, analysis, and data collection activities. We also wish to acknowledge peer reviewers for providing a thoughtful and comprehensive review of an earlier draft of the manuscript.

\section{Author details}

${ }^{1}$ Epidemiology, Oxford Outcomes Ltd., Vancouver, Canada. ${ }^{2}$ Abbvie Inc, North Chicago, IL, USA. ${ }^{3}$ Faculty of Health Sciences, Simon Fraser University, Burnaby, Canada. ${ }^{4}$ Adzoe Inc., Libertyville, USA. ${ }^{5}$ Department of Community Health \& Epidemiology, Dalhousie University, 5790 University Ave., Halifax, Nova Scotia B3H 1V7, Canada.

Received: 5 March 2013 Accepted: 21 March 2014

Published: 5 April 2014

\section{References}

1. World Health Organization: International statistical classification of diseases and related health problems - 10th revision; 2004. http://www.who.int/ classifications/icd/en/.

2. Beck S, Wojdyla D, Say L, Betran AP, Merialdi M, Requejo JH, Rubens C, Menon R, Van Look PFA: The worldwide incidence of preterm birth: a systematic review of maternal mortality and morbidity. Bull World Health Organ 2010, 88:31-38.

3. Petrou S, Mehta Z, Hockley C, Cook-Mozaffari P, Henderson J, Goldacre M: The impact of preterm birth on hospital inpatient admissions and costs during the first 5 years of life. Pediatrics 2003, 112:1290-1297.

4. Wang ML, Dorer DJ, Fleming MP, Catlin EA: Clinical outcomes of near-term infants. Pediatrics 2004, 114:372-376.

5. Huddy $C L$, Johnson A, Hope PL: Educational and behavioural problems in babies of 32-35 weeks gestation. Arch Dis Child Fetal Neonatal Ed 2001, 85:F23-F28.

6. Canadian Institute for Health Information $(\mathrm{CIHI})$ : Too early, too small: a profile of small babies across Canada. Ottawa, Ont.: ClHI; 2009. https://secure.cihi.ca/ free_products/too_early_too_small_en.pdf (Accessed 03/02/2014).

7. Canadian Institute for Health Information (CIHI): Highlights of 2009-2010 selected indicators describing the birthing process in Canada. Ottawa, Ont $\mathrm{CIHl}$; 2011. https://secure.cihi.ca/free_products/Childbirth_Highlights_ 2010-11_EN.pdf (Accessed 03/02/2014).

8. Goldenberg RL, Culhane JF, lams JD, Romero R: Epidemiology and causes of preterm birth. Lancet 2008, 371:75-84.

9. Tucker J, McGuire W: Epidemiology of preterm birth. BMJ 2004, 329:675-678.

10. Saigal S, Doyle LW: An overview of mortality and sequelae of preterm birth from infancy to adulthood. Lancet 2008, 371:261-269.

11. van Baar AL, Vermaas J, Knots E, de Kleine MJ, Soons P: Functioning at school age of moderately preterm children born at 32 to 36 weeks' gestational age. Pediatrics 2009, 124:251-257.

12. Learning Disabities Association of Canada: Learning Disabilities in Canada: Economic Costs to Individuals, Families and Society. Edmonton, Alberta: Learning Disabilities Association of Canada; 2002. http://www.Idalberta.ca/ wp-content/uploads/2010/10/Research_EconomicCosts.pdf.

13. Mangham LJ, Petrou S, Doyle LW, Draper ES, Marlow N: The cost of preterm birth throughout childhood in England and Wales. Pediatrics 2009, 123:e312-e327.

14. Gray D, Woodward LJ, Spencer C, Inder TE, Austin NC: Health service utilisation of a regional cohort of very preterm infants over the first 2 years of life. J Paediatr Child Health 2006, 42:377-383.

15. Hibbard JU, Wilkins I, Sun L, Gregory K, Haberman S, Hoffman M, Kominiarek MA, Reddy U, Bailit J, Branch DW, Burkman R, Gonzalez Quintero VH, Hatjis CG, Landy H, Ramirez M, VanVeldhuisen P, Troendle J, Zhang J: Respiratory morbidity in late preterm births. JAMA 2010, 304:419-425.

16. Stevens TP, Sinkin RA, Hall CB, Maniscalco WM, McConnochie KM: Respiratory syncytial virus and premature infants born at 32 weeks' gestation or earlier: hospitalization and economic implications of prophylaxis. Arch Pediatr Adolesc Med 2000, 154:55-61.

17. Boyce TG, Mellen BG, Mitchel EF Jr, Wright PF, Griffin MR: Rates of hospitalization for respiratory syncytial virus infection among children in medicaid. J Pediatr 2000, 137:865-870.
18. Landry JS, Croitoru D, Jin Y, Schwartzman K, Benedetti A, Menzies D: Health care utilization by preterm infants with respiratory complications in Quebec. Can Respir J 2012, 19:255-260.

19. Kapur V, Basu K: Drug coverage in Canada: who is at risk? Health Policy 2005, 71:181-193.

20. Regie de l'assurance Maladie du Quebec: Statistiques annuelles 1993. Québec City: PQ, RAMQ; 1994.

21. Doyle LW: Neonatal intensive care at borderline viability-is it worth it? Early Hum Dev 2004, 80:103-113.

22. Lee SK, McMillan DD, Ohlsson A, Pendray M, Synnes A, Whyte R, Chien LY Sale J: Variations in practice and outcomes in the Canadian NICU network: 1996-1997. Pediatrics 2000, 106:1070-1079

23. Bolisetty S, Bajuk B, Abdel-Latif ME, Vincent T, Sutton L, Lui K: Preterm outcome table (POT): a simple tool to aid counselling parents of very preterm infants. Aust N Z J Obstet Gynaecol 2006, 46:189-192.

24. Joseph KS, Liu S, Rouleau J, Lisonkova S, Hutcheon JA, Sauve R, Allen AC, Kramer MS: Influence of definition based versus pragmatic birth registration on international comparisons of perinatal and infant mortality: population based retrospective study. BMJ 2012, 344:e746

25. Mangham LJ, Petrou S: Modelling the long-term costs of preterm birth. London, United Kingdom: Tommy's the Baby Charity; 2008.

26. Hardy N: Cost and design analysis of neonatal intensive care units: comparing single family room, double-occupancy, open-bay and combination settings for best design practices, Thesis. Gainesville, Florida: University of Florida; 2005. http://ufdc.ufl.edu/UFE0011780/00001 (Accessed April 2 2014)

27. Narang A, Kiran PS, Kumar P: Cost of neonatal intensive care in a tertiary care center. Indian Pediatr 2005, 42:989-997.

28. Agar JW, Knight RJ, Simmonds RE, Boddington JM, Waldron CM, Somerville CA: Nocturnal haemodialysis: an Australian cost comparison with conventional satellite haemodialysis. Nephrology (Carlton) 2005, 10:557-570

29. Canada S: CANSIM Table 102-4509 - Live births, by birth weight and sex, Canada, provinces and territories, annual; 2009.

30. Canada S: CANSIM Table 102-4512 Live births, by weeks of gestation and sex, Canada, provinces and territories, annual; 2009.

31. Ontario Ministry of Health and Long Term Care: Schedule of Benefits: Physician Services Under the Health Insurance Act; 2012. http://www.health. gov.on.ca/english/providers/program/ohip/sob/physserv/physserv_mn.html (Accessed 03/02/2014).

32. Working in Canada: Median Wage for midwife in Canada; 2012. http://www workingincanada.gc.ca (Accessed 03/02/2014).

33. Ontario Ministry of Health and Long Term Care: Ontario Drug Benefit Formulary; 2012. http://www.health.gov.on.ca/en/pro/programs/drugs/ odbf eformulary.aspx (Accessed 03/02/2014).

34. Ontario Ministry of Health and Long Term Care: Ontario Case Costing Initiative; 2012. http://www.occp.com/mainPage.htm (Accessed 03/02/2014).

35. Harris KC, Anis AH, Crosby MC, Cender LM, Potts JE, Human DG: Economic evaluation of palivizumab in children with congenital heart disease: a Canadian perspective. Can J Cardiol 2011, 27:523-525.

36. Statistics Canada: CANSIM Table 282-0069 Labour force survey estimates (LFS), wages of employees by type of work, National Occupational Classification for Statistics (NOC-S), sex and age group, unadjusted for seasonality, monthly; 2012

doi:10.1186/1471-2431-14-93

Cite this article as: Johnston et al:: The economic burden of prematurity in Canada. BMC Pediatrics 2014 14:93. 